\title{
The Role of Facilitation in Technology-Enhanced Learning for Public Employment Services

\author{
http://dx.doi.org/10.3991/ijac.v7i3.4050
}

\author{
Jenny Bimrose ${ }^{1}$, Alan Brown ${ }^{1}$, Teresa Holocher-Ertl ${ }^{2}$, Barbara Kieslinger ${ }^{2}$, \\ Christine Kunzmann ${ }^{3}$, Michael Prilla $^{4}$, Andreas P. Schmidt ${ }^{5}$ and Carmen Wolf ${ }^{6}$ \\ ${ }^{1}$ University of Warwick, Coventry, UK \\ ${ }^{2}$ Centre for Social Innovation/Technology \& Knowledge, Vienna, Austria \\ ${ }^{3}$ Pontydysgu Ltd, Königsbach-Stein, Germany \\ ${ }^{4}$ Ruhr University of Bochum, Germany \\ ${ }^{5}$ Karlsruhe University of Applied Sciences, Karlsruhe, Germany \\ ${ }^{6}$ Karlsruhe Institute of Technology (KIT), Karlsruhe, Germany
}

\begin{abstract}
Public Employment Services (PES) in Europe are authorities that attempt to match supply and demand on the labor market. Rising unemployment in times of crisis and demographic change are among the main challenges with which PES practitioners, as a direct interface between jobseekers and employers, have to deal. They have to support career adaptability of their clients, as well as to enhance and transform their own individual and collective professional identities, in order to cope successfully with the challenges of a changing labor market. As part of the research project EmployID, we are exploring how to facilitate the learning process of PES practitioners in their professional identity development. The aim of the project is to empower individual PES practitioners, their community, and organizations, to engage in transformative practices, using a holistic tool suite combining e-coaching, reflection, MOOCs, networking, analytical, and learning support tools. The key to successful professional identity transformation is continuous learning. Individuals may take on the role of facilitators for the learning of others as well as being facilitated by peers, technology and environment.
\end{abstract}

Index Terms - Career adaptability, Facilitation, Professional Identity Transformation.

\section{INTRODUCTION}

European Public Employment Services (PES), and their employees, are facing fundamental challenges to the delivery of efficient and effective services, and the need to change their strategies to combat high unemployment, demographic change in increasingly uncertain and dynamic labour markets. Career adaptability, defined as the ability to manage successful transitions in employment, training, education and other contexts, consistently across the life-course, is increasingly seen as a key part of the solution to these challenges [1] [2]. While PES practitioners, nowadays, are supporting the development of career adaptability of their customers/clients, they are faced with changes in their jobs that require them (1) to enhance their own career adaptability and, linked to this, (2) to transform their own individual and collective professional identities. Such a transformational process is a complex and continuous learning process, though resources are often scarce and time for learning is increasingly tight.
The collaborative research project, EmployID, is taking on the challenge to study professional identity transformation processes in European PES with a special focus on the individual, social, and organizational learning aspects involved. An international team of researchers aims to support and facilitate the learning process of PES practitioners in their professional identity development by the efficient use of technologies to provide advanced coaching, reflection, networking, and learning support services. Based on adult learning theories, the project focuses on technological developments that make facilitation services for professional identity transformation cost-effective, and sustainable by empowering individuals, and organizations to engage in transformative practices, using a variety of tools. While most state-of-the-art solutions for supporting learning either directly target individual learning (by making it more effective), or, at the organization's provision of learning opportunities, EmployID particularly focuses on supporting activities that facilitate the learning of others through the use of technology.

This cannot be achieved through a single technological approach, but includes a broad range of solutions that need to complement each other: (1) e-coaching tools that make coaching processes more efficient, and enable the development of coaching skills, (2) tools for reflection and reflexivity that can complement coaching processes (and vice versa, see below), (3) novel networking, and facilitation tools, to support individuals to become effective facilitators for the learning of others, and (4) flexible scorecard visualizations as a form of workplace learning analytics, partially informed by data collected from the user activities and feedback, which enables closed-loop approaches. It also involves sharing of lessons learned amongst PES practitioners, and across PES organizations. This will be achieved through the development and delivery of a Massive Open Online Course (MOOC), which needs to be linked to existing organizational learning and training infrastructures, such as existing learning management systems (LMS).

It is expected that the project results will help PES practitioners to increase their efficiency and effectiveness by adapting to rapidly changing pressures and demands. It is also supposed to support PES organizations in effectively managing the up-skilling of their staff, and delivery of key 
PAPER

The Role of Facilitation In TeChNOlogy-EnHANCEd LEARning FOR PUblic EMPloyment SERVICES

aspects of their services. A comprehensive and empirically validated indicator framework for PES organizations adaptable to their needs shall support the development of a performance improvement culture in the future. The holistic approach of the project (Fig.1) is targeting professional identity transformation on an individual, organizational, and European network level.

In this paper, we present the theoretical background of professional identity transformation (section II). As facilitation of learning and the role of facilitators have been identified to be key for professional identity transformation, we will explore different forms of facilitation, and their conceptual background in section III. In section IV, we present our design approach to developing solutions, and the contextual drivers and barriers that have been encountered (section IV).

\section{PROFESSIONAL IDENTITY TRANSFORMATION}

A core concept driving the implementation of learning innovations in PES is professional identity transformation. Professional identity consists of many different elements that interrelate, as depicted in Fig. 2. First of all, professional identity has an individual and a collective dimension, and for individuals it is linked to other aspects of their personal identities, relating to other life roles. Identity encompasses work activities and work organization, the relationship with other professions, and the professional culture ,as well as processes of becoming part of the profession [3].

Professional work identities are restructured in a dynamic way when employees are challenged to cope with demands for flexibility, changing work situations, and skill needs [3]. Work identities are influenced both by structural factors, and the agency of employers and employees. Today's PES practitioners, and employees more generally, are increasingly required to exhibit flexibility at work, and are challenged to engage with work that is itself changing in significant ways. As their roles change, so do their identities, with these changes requiring developmental attitudes to learning and work to enable practitioners to engage actively in shaping their changing work processes, with work identities playing a decisive role in this process [4]. As well as being shaped by the organization and individuals, work identities are also influenced by attachments to specific work groups [5], or wider communities of practice [6]. All these processes change over time, as can the significance individuals ascribe to them [3] [7].

In this research it is anticipated that, at the individual level, emerging new demands, with their implications for shifting skill needs, generate a potential for conflict with traditional work orientations, and associated values, norms, work ethics, and work identity patterns of employees. One focus of the development will therefore be supporting individuals' strategies for dealing with such conflicts. The overall argument is that both PES practitioners and their clients need support as their work-related identities become increasingly unstable and disrupted [8]. The key question is, what role can ICT supported learning and skills development play in better equipping individuals to handle instabilities and uncertain working conditions through professional identity transformation?

Ultimately, any identity formation process is an achievement of the individual. The process of acquiring a work identity takes place within particular communities

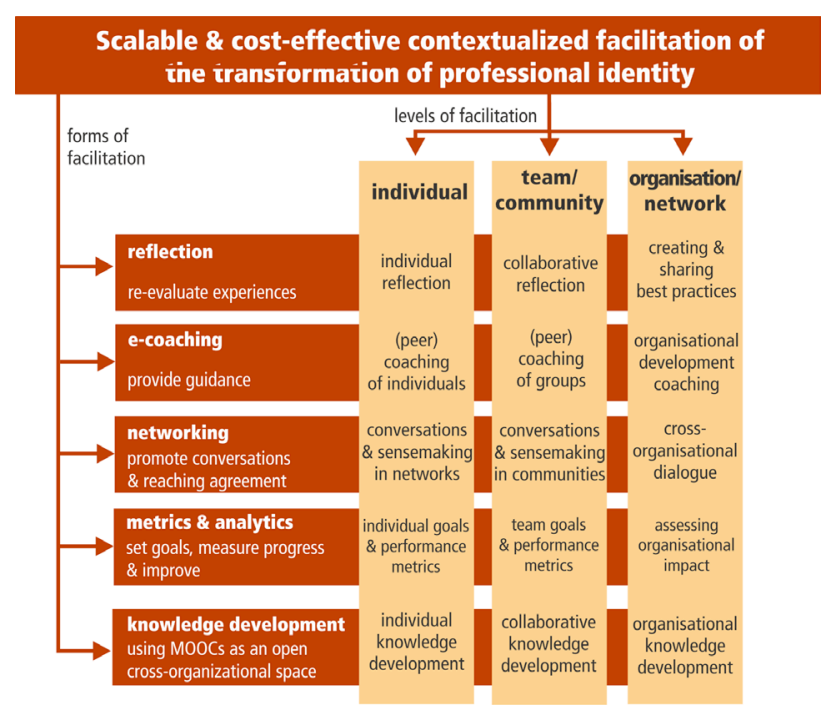

Figure 1. EmployID approach

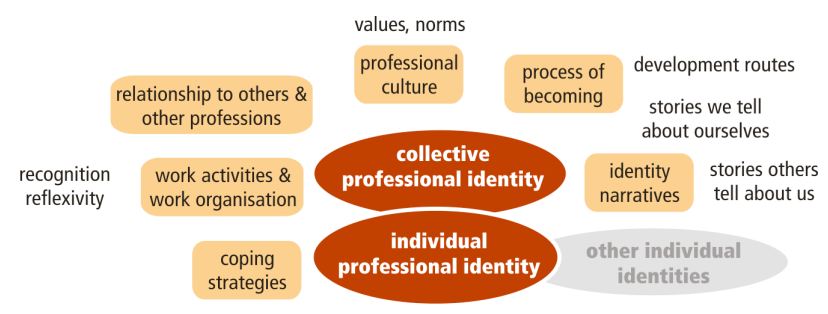

Figure 2. Elements of Professional Identity

where socialization, interaction, and learning are key elements. However, it is not simply a matter of taking on identities and roles, which are pre-existent and prestructured, rather, individuals can also take a proactive role in actively reshaping the community of practice [3]. Hence there is scope for individual agency to act upon the structures and processes. Work identities need to be understood in a dynamic way. Individuals are active participants in the creation of "new" communities of practice [9] [10] [11], and the use of "super-users", and ICT-supported facilitation services, and processes are the distinctive and innovative drivers of the EmployID project in this respect.

PES practitioners increasingly need to develop multidimensional (individual and collective) occupational identities to cope with socio-economic and technological change [12]. A focus upon development of individual and collective skills, knowledge, competencies and active career development, using commitments to learning, continuing vocational training, mobility, and flexibility is an important means to achieve this. This shift in skills requirements is underpinned by the increased importance of communications skills, a willingness to engage in learning and reflexivity; while reflection on experience over time may be particularly significant in the build-up of implicit or tacit knowledge as well as explicit knowledge [13]. Feedback and support can be important too, in supporting role change and identity development.

A further challenge for professional identity transformation relates to the problems faced in the deployment of knowledge. A coach can play an important role in helping individuals re-contextualize what they have learned and help them think and reflect both explicitly and implicitly about what constitutes effective performance in a changing context [14]. 


\section{FACILITATION}

The term "facilitation" has been used since the second half of the 20th century in many fields (e.g. organizations, education, therapy, mediation, research). Facilitation derives from the Latin word "'facilis", which means "easy to do, and is, according to [15] "one of the most misunderstood and abused terms used in management" [16]. A quite unambiguous definition for facilitation describes it as "a technique by which one person makes things easier for others" [17]. This very open definition lacks acknowledgment of the complexity of facilitation and facilitator roles. As explained in [18], facilitator is to be viewed as an umbrella concept with its variety in roles, and different combinations of roles and tasks.

\section{A. Conceptual approaches to facilitation}

A group of scholars have started to study the role of facilitation (under the term of "guidance") for collective knowledge development in several case studies [19]. This work has led to the identification of guidance activities where organizations with successful support for collective knowledge development excel others. The range of activities include, e.g., providing feedback, irritating \& challenging, structuring \& organizing, encouraging, evaluating $\&$ assessing results, or rewarding, among others. Thus, facilitating the learning of others takes a variety of forms, which range from stimulating reflection and elements of coaching processes via leadership activities, to influencing motivation, and designing incentive structures.

Accordingly multiple perspectives on facilitation unfold, which set the scene for EmployID:

- Humans as facilitators. As part of formal and informal learning, individuals take over various roles in supporting the learning of others, ranging from classical trainers with a strict separation of roles between learners and facilitators, via coaches or moderators of group learning processes to peer learning situations with frequently changing roles between peers. Here the question for EmployID is: How can we make facilitators more efficient so that more individuals can benefit from facilitation, especially as training or coaching can be rather costly forms?

- Tools as facilitators. Technology is not only used to support facilitators, but can do facilitation itself. For informal learning situations, this is being investigated, e.g., under the label of "scaffolding" [20], where scaffolding targets at building learning networks, and creating and using digital materials.

- Environments as facilitators and individuals as facilitators for environments. The most neglected aspect of facilitation is the role of the surrounding environment. In the case of workplace learning, the most important factor can be the organizational culture. Its incentive structures, and the economies of cooperation heavily influence motivation and can pose barriers to creativity and openness to change [21]. But also leadership roles (both formal and informal) are often underestimated in their effect, such as those by claimants or change agents [22]. Support in this area ranges from systematic design processes that are participatory and include motivational aspects [23] to workplace learning analytics solutions.

\section{B. Roles of human facilitators}

The role of the facilitator is concerned with supporting the performance of specific tasks while interacting with the group, which leads to the facilitator having multiple roles [18]. Essential functions of a facilitator are "to create and sustain an environment in which a group can accomplish its tasks and learn about itself in the process" [24]. According to the International Association of Facilitators, a facilitator can thus be seen as "someone who uses knowledge of group processes to formulate and deliver the needed structure for meeting interactions to be effective. The facilitator focuses on effective processes (meeting dynamics) allowing the participants to focus on the content or the substance of their work together"1.

A qualitative study on facilitation in Group Support Systems (GSS) (1993) makes clear how important certain skills of a facilitator are, by showing the educational background of the 37 interviewed facilitators. They are mainly educated formally on topics such as organizational psychology, organizational development, behavioral studies etc. [24]. Skills that are important for the facilitator include: flexibility, the ability to listen and understand, the abilty to empathize with individuals as well as groups, self-awareness; and the ability to remain neutral [24].

Beranek et al. [24] argue that there is often not much high-quality training available for facilitation, so facilitators in EmployID will be trained on the facilitation roles that are identified during the project. Possible facilitation roles could be linked to the main topics of the project: ecoaching (intervision \& self-coaching), creativity, reflection, technology use and networking activities.

Aiming to increase the career adaptability of PES practitioners and their clients, this carries the notion of individuals' active engagement with learning and exerting individual agency to shape their work biographies, and individual careers. Individuals are expected to take an active role in the continual updating and directing of their skills and competences, which demands a range of abilities, which are constructed around "self", including attributes such as self-monitoring, self-realization, selfsocialization, or self-initiative.

But not all individuals can become the "designers" of their individual careers to the same degree. It depends on their level of expertise, passion, and also creativity, of the individual. These aspects are not only relevant when designing one's own career, but also when adapting one's work tasks to the changes from outside in innovative ways. In general four levels of creativity can be observed in people's lives: doing, adapting, making, and creating [25] as indicated in Table 1.

TABLE I.

LEVELS OF PERSONAL CREATIVITY

\begin{tabular}{|c|l|l|}
\hline Level & \multicolumn{1}{|c|}{ Motivation } & \multicolumn{1}{c|}{ Requirements } \\
\hline Doing & $\begin{array}{l}\text { To get something done, to } \\
\text { be productive }\end{array}$ & $\begin{array}{l}\text { Minimal interest } \\
\text { Minimal domain expertise }\end{array}$ \\
\hline Adapting & $\begin{array}{l}\text { To make something my } \\
\text { own }\end{array}$ & $\begin{array}{l}\text { Some interest } \\
\text { Some domain expertise }\end{array}$ \\
\hline Making & $\begin{array}{l}\text { To assert my ability or } \\
\text { skills, make something } \\
\text { with "my own hands" }\end{array}$ & $\begin{array}{l}\text { Genuine interest } \\
\text { Domain experience }\end{array}$ \\
\hline Creating & $\begin{array}{l}\text { To express my creativity, } \\
\text { to feel inspired }\end{array}$ & $\begin{array}{l}\text { Passion } \\
\text { Domain expertise }\end{array}$ \\
\hline
\end{tabular}

${ }^{1}$ http://www.iaf-world.org/Libraries/Facilitation_Articles/ASQIAF_Facilitation_Primer.sflb.ashx 
PAPER

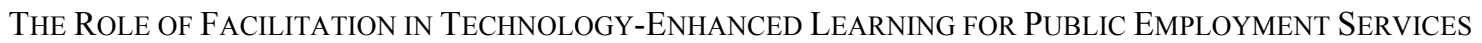

In becoming facilitators, individuals are faced with the question about how to offer relevant experiences to facilitate people's expression of creativity on all levels. According to [26] facilitators need to:

- Lead people who are on the "doing" level of creativity,

- Guide those who are at the "adapting" level,

- Provide scaffolds that support and serve people's need for creative expression at the "making" level (A scaffold is a special type of communicational space, one that supports and affords creative behavior.), and

- Offer a clean slate for those at the "creating" level

\section{Facilitation via tools (e-coaching and reflection)}

In EmployID facilitation not only takes place through the methods used by the identified facilitators in their specific facilitator roles, but will also be supported or conducted by the technology used to facilitate individual, group and organizational development.

For e-coaching there are two major tasks: the development of a self-e-coach tool for self-coaching; and a tool for peer coaching (intervision) that will be referred to as eintervision. Considering sustainability and costeffectiveness of coaching in PES organizations, it is unrealistic to assume that professional coaching can support PES practitioners on-site to a large extent. Self-e-coaching support seems to be a good alternative. The self-e-coach will be pre-structured and formalized, and will therefore facilitate self-coaching without the need of support from other staff with coaching skills. Within the e-intervision concept there are different roles to be accepted by the group members (e.g., client, coach, counsellors) with different tasks that are to be facilitated by the e-intervision tool, e.g. the process controlling, the use of "powerful" coaching questions, the managing of the different roles. The method will be taught in training on peer coaching (intervision), since for some of the roles coaching skills are required, to a certain degree. Through working with ecoaching, and learning about the training method, it is expected that PES practitioners become facilitators of their own learning, their peers, and their clients. "Research shows that under certain conditions, groups using collaboration technologies such as group support systems (GSS) can gain substantial improvements in the effectiveness and efficiency of their work processes" [18].

Reflection support facilitates learning on work by enabling individuals and groups to identify good and bad practices as well as the rationales behind them, and opportunities to improve their own practice. This is done by a process of going back to past experiences (including emotions during these experiences), reassessing these experiences in the light of current knowledge, and deriving insights for future work from this [27]. Reflection may then be done by an individual or by groups, in which an individual experience or issue may be complemented by similar experiences of others, and their perspectives on it. A facilitator may structure this process by asking questions, triggering or guiding reflection [28], and by ensuring that reflection participants can bring in their perspectives on the issue reflected upon [29]. Relying on a facilitator, though, has downsides in reflection support, as the facilitator can only be active in situations in which people explicitly step back from work to engage in reflection, such as in project debriefings.
In practice, reflection happens mostly during work, and the time to explicitly step back is either not given or available too late, when (parts of) the experience has been forgotten [30]. Tools have therefore been found to keep experiences vibrant, by writing them down in journals [31], or keeping pictures [32], by prompting people to reflect on these experiences [33] [34], and by enabling people to reflect over time [30]. For PES practitioners, there needs to be the means to reflect individually, e.g. by comparing their own performance with others, and the means to reflect together in order to create ideas and insights on how to change, and what this change will mean for their work.

Reflection and coaching may complement each other, as they are tackling a similar area of explorative, informal learning at work. Coaching interfaces with learning via given processes that participants can go through with their coaches or tools, and reflection supports it by sustaining experiences, and creating a space for reflection in which participants can reflect over time [30]. Insights from reflection may thus support the coaching process and vice versa - by combining facilitation and tools for both approaches, we can create synergies and enable people to choose the method they perceive to be adequate for their given problem. For example, if individuals seek to clarify why their job performance is below average, they may choose to run a coaching process enabling them to come up with insights on this, or they may choose to initiate a process of reflection with their colleagues to find opportunities to improve. Besides this, e-coaching may be used to make PES practitioners more reflective, that is, coach them in reflecting individually, and together as well as showing them how to help others in reflection, becoming a reflection facilitator.

\section{Environments as facilitators}

Organizational culture, working autonomy, social norms, social relationships, legitimated hierarchies etc. are factors that may impact the extent to which individuals are ready actively, as well as sustainably, to share and seek knowledge, and to overcome learning barriers. Empirical research in the field of organizational learning has revealed several factors that strongly influence the collaborative knowledge sharing behavior of individuals in organizations.

Norms of cooperation and helping each other, aspects of involvement and consistency, stimulate knowledge sharing behavior, while bureaucratic hierarchies and competition might considerably hamper open exchange and sharing. The more an employee believes that information sharing is a correct and socially expected workplace behavior, the more he or she will contribute or seek knowledge. Active support from the management in such activities via resources, time, recognition, and clear visions and guidelines, can facilitate learning in this regard [35], and needs to be taken into consideration when talking about a facilitation framework for European PES.

Existing studies show that socio-technical issues in organizational environments especially have to be taken into consideration when implementing learning tools that support reflection, coaching and networked learning [30]:

- The introduction of tools and processes for reflection and e-coaching changes the way knowledge and insights are created as well as the control of this pro- 
PAPER

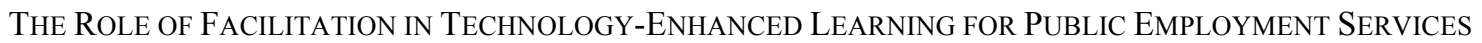

cess from a managerial task to a bottom-up task, driven by workers [30]. Workers start bringing up their own solutions and ideas on how to change work, involving facilitators, but possibly without involving management in the early phases of solution or idea development. This needs to be acknowledged and supported actively by managers, who need to see such change as a necessary complement to existing processes, rather than as a loss of control and management power.

- Reflection and coaching take time. Although tools can support workers to reflect whenever there is time for this, instead of requiring them to step back from work immediately, workers still need to document issues, and communicate with others on these issues. This causes two barriers on the socio-technical level. First, workers need to see the value added by using reflection tools, as otherwise they will not spend their scarce time on these tools. Second, reflection needs to be explicitly encouraged by management to be perceived as part of work, rather than extra effort.

- Reflection and coaching tools need to fit the organizational context, as well as individual, and group work practices. This includes the need to embed tools, processes, and facilitation into existing work processes, and to use technologies and devices that are accessible, and can be used at work. In particular, previous research found the integration of reflection tools into meetings, and the participation of managers in reflection conducted with these tools (e.g., driving and facilitating it) to be helpful [36].

\section{Design Challenges}

\section{A. Methodological approach}

In order to align this research closely to the needs of organizations and individual practitioners a participatory design approach has been chosen for the development of facilitation services. In participatory design, end-users cooperate with researchers and developers in all aspects of the innovation process, from initial exploration and problem definition via design and implementation, through to evaluation.

Fostering the mutual exchange of practical context knowledge from PES practitioners with the more systematised, de-contextualised knowledge of researchers, the project specifically targets and makes use of selected groups of facilitators in PES. These key persons are guided to cascade the process of innovation, including the introduction and development of new learning processes, and knowledge products throughout their organizations. They act as facilitators towards their peers by providing them with scaffolds, directing their attention to relevant materials, and supporting their learning processes. In addition, these facilitators are key informants for the research team, and provide valuable feedback during the design and development process.

Adopting an action research approach, the design activities will alternate "between practical work in the field to support the desired changes, and systematic data collection and analysis of the practical work with the aims of improving the action and contributing to theory building." [37] While the practical work in the field will support PES practitioners' transformation processes, the theoretical work will contribute to a conceptual model on "Professional Identity Transformation Facilitation".

One of the first challenges in this iterative design process is to consolidate the diverse information requirements from the researchers' perspective, and develop a set of flexible data collection instruments, which can be adapted to the different contextual constraints of PES, such as limited effort and time, privacy regulations, organizational preferences etc.

\section{B. Contextual challenges}

When entering the complex working environments of European PES a broad variety of contextual challenges start to unfold. While PES exist in all European countries, they differ significantly in terms of their internal governance structures, as well as their services offered to the public. In some countries there has been a shift from working with job seekers in face-to-face meetings to offering a purely online service to their clients. Other countries have shifted their focus from individual job seekers to employers, and offer specific services for hiring organizations. Demographic change and economic developments are just two external factors driving these pubic organizations to innovate their services and their internal structures. Latest developments in ICT, as well as the possibilities offered by big data, are likewise influencing changes in these organizations.

PES in Europe have an important function for society. They are a public authority offering a service to the community and the economy at the same time. Thus they play a key role in the European labor market, and are highly visible. However, in some countries PES do not necessarily enjoy the best reputation amongst the general public, and are currently seeking ways to improve their public perception.

Within this complex context of environmental challenges PES are seeking guidance in their change processes on an organizational level, as well as on an individual practitioner level. In order to support the personal learning and career management of individual practitioners, a shift towards a culture of openness, and towards a learning organization has to take place within certain constraints defined by e.g. the regulatory framework.

From a project perspective the challenges are equally complex, but manifest themselves on different levels. During the initial exploration phase identified challenges can be allocated to andragogical/conceptual issues, technical issues, socio-technical issues, and methodological issues:

Andragogical/conceptual issues:

- Professional identities are developed based on a set of defined competences and skills, and also on the more implicit assumptions of performance expectations and work attitudes. A learning facilitation model would thus needs to link formal learning processes and competency schemes existent in PES with informal processes of knowledge exchange, performance, and attitude changes relevant for identity transformation.

- To help European PES to cope with labor market challenges a facilitation framework needs to support the innovation capacity found broadly amongst individual PES practitioners in Europe, while at the same time complying with standardized processes and 
rules defined differently for each of the national PES. This potential conflict between innovation and regulation is also reflected in professional identity transformation processes adding additional requirements for any new concepts and tools.

- Another issue is balancing depth and breadth of the identity transformation model, to be detailed enough to provide clear guidance on how to successfully facilitate transformative processes in todays' public services, while at the same time being adaptable to the different contexts of European PES.

Technological issues:

- Current learning environments have been developed for national PES, and consist of a portfolio of tools and learning management systems (LMS) that support rather top-down, formal-skill acquisition processes. Complementing the existing learning processes in these systems with innovative facilitation tools would require an opening up of these systems. Ideally, a new technological solution would provide a "one-stop-shop" for learners in the form of an integrated solution.

- Conflicts may arise between the need for confidentiality in organizational networks of PES, and the need to openly access and share learning content. Strict regulations, which aim to protect the privacy of employees and clients, might further contribute to this challenge.

Socio-technical issues:

- PES practitioners in different European countries follow a clear set of competence requirements, continually adapted to changing labor market needs, that are strongly managed top-down. With the implementation of collaborative learning, reflection and coaching tools, the way knowledge and best practice is created changes, and becomes a bottom-up process driven by workers themselves. These bottom-up innovation processes require support and acknowledgement from management.

- Time for reflection, coaching, and collaborative learning is essential, but one of the most scarce resources for PES practitioners. The time needed to document experiences, and discuss them with peers, as well as the time to participate in coaching processes, needs to be integrated in daily routines and processes. Collaborative reflection should become a standard part of team meetings, and individual reflection and coaching should be an officially appreciated task on PES practitioners' agendas.

Methodological issues:

- Participatory design research requires strong commitment from all parties involved. While there is clear commitment from all sides in this process, the resources tend to be scarce in PES. Thus limited time for the continuous involvement in the research project might become an issue.

- Expectation management on different levels is another important challenge for the project as expectations from the management point of view might differ from the PES practitioners' perspective.

- Bringing people into a collaborative design process most beneficial to their ability to participate, researchers will need to bring in theories on learning and identity transformation in a way which can be handled by the whole interdisciplinary team. This not only touches aspects of finding a common language, but also breaking down theoretical concepts to the practical requirements and contexts of European PES.

Fig. 3 below illustrates the described challenges for implementing learning facilitation processes in European PES. It requires a combinatory effort to link diverging as well as complementary expectations, constraints and cultures.

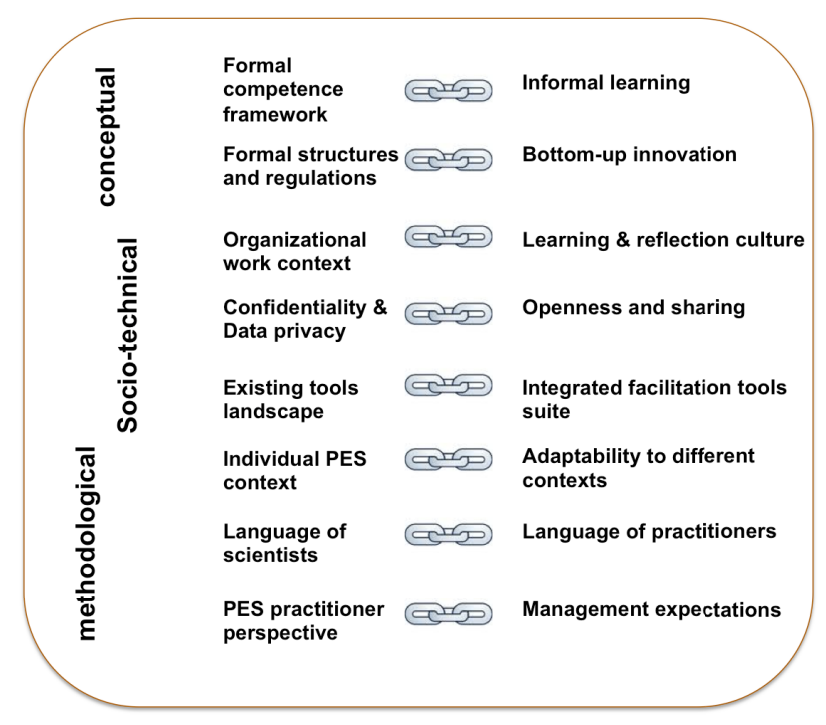

Figure 3. Contextual Challenges for facilitating learning innovation in PES

\section{CONCLUSIONS}

Professional identity transformation processes are becoming increasingly important in today's workplaces, and career adaptability is often required from employees across sectors, in private industries, as well as in public administration. The ability to manage one's career and its related continuous learning becomes an important competence for any individual participating in the labor market.

In order to support individuals in their career adaptability, and professional identity transformation facilitation on an individual, group and organizational level appears to be a key process. Facilitation, and the associated roles of facilitators, are, however, not trivial, as it requires a combination of multiple perspectives: (i) humans as facilitators, (ii) tools as facilitators, and (iii) environments as facilitators. In each of these areas, promising prior work to build upon exists, but it needs to be further developed and combined into a holistic concept to meet the challenges encountered by European PES.

From the perspective of "humans as facilitators" it requires a better understanding of which facilitation roles are needed to support the diversified transition processes of PES practitioners, and how to support these facilitation roles. A major challenge is to define a set of facilitation support activities that can address the diversified needs of individuals with different expertise, interests, learning styles, and identities in the very heterogeneous contexts of European PES. 
Using "tools as facilitators" faces the challenge of integration of the facilitation tool suite in a landscape of existing tools, again different in each European PES. An important question is also, how to consider issues of data privacy and security, while implementing tools for openness and sharing.

Looking at the environment we are challenged to link bottom-up innovation, and informal learning processes to different organizational work and learning contexts, as well as formal structures and regulations. We expect motivational drivers and barriers for collaborative learning with, and by the help of others to play a key role here. Furthermore, acceptance in an increasingly performanceoriented context requires workplace learning analytics approaches that help organizational sense-making of learning activities and their effects.

These challenges require very careful explorations of the organizational structures, and the regulatory systems in which these organizations operate, as well as the motivations, drivers, and barriers on the side of the individuals.

\section{ACKNOWLEDGMENT}

EmployID" "Scalable \& cost-effective facilitation of professional identity transformation in public employment services" is a research project supported by the ICT program of the European Commission under the $7^{\text {th }}$ Framework Program (project no. 619619).

The authors thank the whole project consortium for their input and commitment to the project.

\section{REFERENCES}

[1] Bimrose, J., \& Hearne, L. (2012). Resilience and Career Adaptability: Qualitative Studies of Adult Career counselling. Journal Of Vocational Behavior, Vol. 81, No. 3, pp.338 - 344. http://dx.doi.org/10.1016/j.jvb.2012.08.002

[2] Brown, A., Bimrose, J., Barnes, S-A. \& Hughes, D. (2012). The role of career adaptabilities for mid-career changers. Journal of Vocational Behavior, Vol. 80, No. 3, pp. 754 - 761 http://dx.doi.org/10.1016/j.jvb.2012.01.003

[3] Brown, A. (1997). A dynamic model of occupational identity formation. In: Brown, A. (ed.) Promoting Vocational Education and Training: European Perspectives. Tampere: University of Tampere, pp. 59-67.

[4] Heinz, W.R. (2003). From work trajectories to negotiated careers: The contingent work life course; in: J.T. Mortimer \& M.J. Shanahan (eds.): Handbook of the Life Course. New York: Kluwer/Plenum, pp. 185 - 204. http://dx.doi.org/10.1007/978-0306-48247-2_9

[5] Baruch, Y. \& Winkelmann-Gleed, A. (2002). Multiple commitments: a conceptual framework and empirical investigation in a Community Health Services Trust, British Journal of Management, Vol. 13, No. 4, pp. 337-357. http://dx.doi.org/10.1111/ $\underline{1467-8551.00250}$

[6] Lave, J., \& Wenger, E. (1991). Situated learning: Legitimate peripheral participation. Cambridge: Cambridge University Press. http://dx.doi.org/10.1017/CBO9780511815355

[7] Ibarra, H. (2003). Working Identity: Unconventional Strategies for Reinventing Your Career. Boston, MA: Harvard Business School Press.

[8] Carruthers, B., \& Uzzi, B. (2000). Economic sociology in the new millennium. Contemporary Sociology, Vol. 29, No. 3, pp. 486494. http://dx.doi.org/10.2307/2653936

[9] Lave, J. (1993). The practice of learning. In S. Chaiklin and J. Lave (eds). Understanding practice - Perspectives on activity and context. Cambridge: Cambridge University Press, pp. 3 -32. http://dx.doi.org/10.1017/CBO9780511625510.002

\footnotetext{
${ }^{2}$ http://employid.eu/
}

[10] Wenger, E. (1999). Communities of practice: Learning, meaning, and identity. Cambridge: Cambridge University Press

[11] Billett, S. (2004). Learning through work: Workplace participatory practices. In H. Rainbird, A. Fuller \& A. Munro, Workplace learning in context. London: Routledge, pp.109-125.

[12] Kirpal, S. (2004). Researching work identities in a European context, Career Development International, Vol. 9, No. 3, pp. 199 - 221. http://dx.doi.org/10.1108/13620430410535823

[13] Eraut, M. (2000). Non-formal Learning and Tacit Knowledge in Professional Work. British Journal of Educational Psychology, Vol. 70 , No. 1, pp. 113 - 136. http://dx.doi.org/10.1348/ 000709900158001

[14] Evans, K., Guile, D. (2012). Putting different forms of knowledge to work in practice. In: J Higgs, R Barnett, S Billett, M Hutchings \& F Trede (eds). Practice-Based Education: Perspectives and Strategies. Rotterdam: Sense Publisher. http://dx.doi.org/10.1007/ 978-94-6209-128-3 9

[15] Zimmerman, A.L., Evans, C. J. (1993). Facilitation-- from discussion to decision. East Brunswick, N.J: Nichols.

[16] Hogan, C. (2012). Understanding Facilitation: Theory \& Principles. London: Kogan Page.

[17] Kitson A., Harvey G. \& McCormack B. (1998). Enabling the implementation of evidence-based practice: a conceptual framework. Quality in Health Care Vol. 7, pp. 149-158. http://dx.doi.org/10.1136/qshc.7.3.149

[18] Kolfschoten, G. L.. Niederman, F., Briggs, R.O. \& de Vreede, G.J. (2012). Facilitation Roles and Responsibilities for Sustained Collaboration Support in Organizations. Journal of Management Information Systems, Vol. 28, No. 4, pp. 129 - 161. http://dx.doi.org/10.2753/MIS0742-1222280406

[19] Kaschig, A., Maier, R., Sandow, A., Lazoi, M., Schmidt, A., Barnes, S.A., Bimrose, J., Brown, A., Bradley, C., Kunzmann, C., Mazarakis, A. (2013). Organizational Learning from the Perspective of Knowledge Maturing Activities, IEEE Transactions on Learning Technologies, Vol. 6, No. 2, pp. 158-176, http://dx.doi.org/10.1109/TLT.2013.14

[20] Ley, T., Cook, J., Dennerlein, S., Kravcik, M., Kunzmann, C., Laanpere, M., Pata, K., Schmidt, A., Purma, J., Sandars, J., Santos, P. (2013). Scaling Informal Learning: An Integrative Systems View on Scaffolding at the Workplace. In D. Hernández-Leo, T. Ley, R. Klamma, \& A. Harrer (Eds.), Proceedings of the 8th European Conference on Technology-enhanced Learning (pp. 484489). Heidelberg: Springer.

[21] Kunzmann, C., Schmidt, A., Braun, V., Czech, D., Fletschinger, B., Kohler, S., Lüber V., (2009). Integrating Motivational Aspects into the Design of Informal Learning Support in Organizations. In: 9th International Conference on Knowledge Management and Knowledge Technologies, September 2-4, 2009, Graz, Austria,

[22] Kaschig, A., Maier, R. \& Sandow, A. (2011). Effects of interventions into improving knowledge maturing. In S. N. Lindstaedt \& M. Granitzer (eds.), I-KNOW (p./pp. 6), ACM. ISBN: 978-14503-0732-1

[23] Holocher-Ertl, T., Kunzmann, C., Müller, L., Rivera-Pelayo, V., Schmidt, A. (2013). Motivational \& Affective Aspects in Technology Enhanced Learning: Topics, Results, and Research Route In: ECTEL 2013, Springer.

[24] Beranek, P., Beise, C. \& Niedermann, F. (1993). Facilitation and Group Support Systems. Proceedings of the Twenty-Sixth Annual Hawaii International Conference on Systems Sciences, Vol. 4, pp. 199-207. http://dx.doi.org/10.1109/HICSS.1993.284182

[25] Sanders, E. B.-N. (2006). Scaffolds for building everyday creativity. In J. Frascara (Ed.), Design for Effective Communications: Creating Contexts for Clarity and Meaning. New York, NY: Allworth Press.

[26] Sanders, E. B. N., \& Stappers, P. J. (2008). Co-creation and the new landscapes of design. Co-design, Vol. 4, No. 1, pp. 1-16. http://dx.doi.org/10.1080/15710880701875068

[27] Boud D. (1985). Reflection: Turning experience into learning. London: Kogan Page.

[28] Daudelin M. W. (1996). Learning from experience through reflection. Organizational Dynamics, Vol. 24, No. 3, pp. 36-48. http://dx.doi.org/10.1016/S0090-2616(96)90004-2 
[29] Dyke M. (2006). The role of the "Other" in reflection, knowledge formation and action in a late modernity. International Journal of Lifelong Education, Vol. 25, pp.105-123. http://dx.doi.org/10.1080/02601370500510728

[30] Prilla M., Pammer V., Krogstie B. (2013). Fostering Collaborative Redesign of Work Practice: Challenges for Tools Supporting Reflection at Work. Proceedings of European Conference on Computer Supported Cooperative Work. ECSCW 2013.

[31] Scott S. G. (2010). Enhancing Reflection Skills Through Learning Portfolios: An Empirical Test. Journal of Management Education, Vol. 34, pp. 430-457. http://dx.doi.org/10.1177/105256290 9351144

[32] Fleck R., Fitzpatrick G. (2010). Reflecting on reflection: framing a design landscape. Proceedings of. 22nd Conference on Computer.Human Interaction Special Interest Group, Australian ComputerHuman Interaction. ACM, Brisbane, Australia, pp 216-223

[33] Isaacs E., Konrad A., Walendowski A., et al. (2013). Echoes from the past: how technology mediated reflection improves well-being. Proceedings of SIGCHI Conference on Human Factors Computer Systems. ACM, Paris, France, pp 1071-1080. http://dx.doi.org/10.1145/2470654.2466137

[34] Thillmann H., Künsting J., Wirth J., Leutner D. (2009). Is it Merely a Question of "What" to Prompt or Also "When" to Prompt? Z Für Pädagog Psychol, Vol. 23, pp. 105-115. http://dx.doi.org/10.1024/1010-0652.23.2.105

[35] Holocher, T., Kieslinger, B., Fabian, C.M. (2011). Applying Participatory Methods to Address Motivational Aspects in Informal Workplace. International Journal of Advanced Corporate Learning, Vol 4, No.1. http://dx.doi.org/10.3991/ijac.v4i1.1412

[36] Prilla M. (2014). User and Group Behavior in Computer Support for Collaborative Reflection in Practice: An Explorative Data Analysis. COOP 2014 - Proceedings of the $11^{\text {th }}$ International Conference on the Design of Cooperative Systems.

[37] Clement, A., \& van den Besselaar, P. (1993). A retrospective look at PD projects. Communications of the ACM, 36(4), 29-37. http://dx.doi.org/10.1145/153571.163264

\section{AUTHORS}

Prof. Jenny Bimrose works at the Institute for Employment Research, University of Warwick, Coventry, England. (e-mail: jenny.bimrose@warwick.ac.uk).
Prof. Alan Brown works at the Institute for Employment Research, University of Warwick, Coventry, England. (e-mail: alan.brown@warwick.ac.uk).

Teresa Holocher-Ertl M.A., is with the Technology \& Knowledge Unit, Centre for Social Innovation, Vienna, Austria (e-mail: holocher@zsi.at).

Dr. Barbara Kieslinger is with the Technology \& Knowledge Unit, Centre for Social Innovation, Vienna, Austria (e-mail: kieslinger@zsi.at).

Christine Kunzmann works as a researcher for Pontydysgu Ltd. (e-mail: kontakt@christinekunzmann.de).

Dr. Michael Prilla works in the Information and Technology Management group of the Institute of Work Science at the University of Bochum, Germany (e-mail: michael.prilla@rub.de).

Prof. Andreas Schmidt is with the Institute for Learning and Innovation in Networks, Karlsruhe University of Applied Sciences (e-mail: andreas_peter.schmidt@hs-karlsruhe.de)

Carmen Wolf, M.A., is with the Research Group Engineering Didactics, Institute for Vocational Education and Training, Karlsruhe Institute of Technology (KIT) in the State of Baden-Wuerttemberg, Karlsruhe, Germany (email: carmen.wolf@kit.edu).

This work was supported in part by the European Union's Seventh Framework Programme for research, technological development and demonstration under grant agreement no. 619619. It is an extended and modified version of a paper presented at ICELW 2014, the Seventh Annual International Conference on E-learning in the Workplace, held from June 11-13, 2014 in New York. Submitted 15 July 2014. Published as resubmitted by the authors 14 Octoner 2014. 